\title{
Clinical outcome of sodium alginate therapy in radiation-induced pharyngeal mucositis: experience of a single Japanese institution
}

\author{
Shinsaku Yamaguchi®
}

\begin{abstract}
Purpose: We investigated the clinical outcome of sodium alginate treatment in radiation-induced pharyngeal mucositis (RIPM) after neck irradiation.

Materials and methods: The study population included 32 patients (11 lung cancer, 10 breast cancer, 7 head and neck cancer, and 4 other primary lesions) who underwent neck external beam radiotherapy at the authors' institution between June 2006 and 2016. The patients received 5\% sodium alginate solution orally for RIPM. Those who were followed up for less than 2 months or did not receive $5 \%$ sodium alginate were excluded from this retrospective study. RIPM was graded weekly as an acute toxicity according to the Common Terminology Criteria for Adverse Events (CTCAE), version 4. The administration of 10-15 ml of sodium alginate before each meal was continued until the radiotherapy was completed and after resolution of odynophagia. The efficacy of sodium alginate was assessed by two radiation oncologists as follows: Grade I, ineffective; grade II, moderately effective; grade III, very effective. When sodium alginate was ineffective, other analgesics, such as nonsteroidal antiinflammatory drugs (NSAIDS) or opioids, were added. Relationships between the presence/absence of additional analgesics and the radiation dose were investigated.
\end{abstract}

Results: The median duration from the start of irradiation to sodium alginate administration was 15 days (range, 536 days). RIPM improved in 29/32 patients (grade: $\|, n=22 ;\| I, n=7$ ). Three patients showed no improvement. No sodium alginate-related toxicities occurred. Additional analgesics were required in 5/32 patients. The radiation dose in these 5 patients was significantly higher than that in the sodium alginate-alone group ( $63.6 \pm 7.8 \mathrm{~Gy}$ vs $48.3 \pm 14.8 \mathrm{~Gy}$, $P=0.02$ ). Patients who received $>50$ Gy tended to require additional analgesics more frequently than those who received $\leq 50$ Gy $(P=0.10)$.

Conclusions: The concurrent administration of sodium alginate and neck irradiation was feasible and tolerable without obvious toxicities. Under certain conditions sodium alginate could be a promising alternative to NSAIDs and opioids in RIPM. The results justify further prospective evaluations with detailed treatment protocols to clarify whether sodium alginate can improve RIPM.

Keywords: Radiation induced pharyngeal mucositis, Neck irradiation, Sodium alginate, Pharyngeal mucositis 


\section{Introduction}

Radiation-induced pharyngeal mucositis (RIPM) is a common, painful, debilitating side effect of neck radiotherapy (RT). Severe RIPM is associated with adverse effects on quality of life, weight loss, increased opioid use, gastrostomy-dependent feeding, and an increased hospitalization period. Additionally, prolongation of the overall radiation time has an adverse effect on the radiocurability of head and neck squamous cell carcinoma [1-3]. There are no definite guidelines on the management of RIPM, but the basic strategy is to use nonsteroidal anti-inflammatory drugs (NSAIDs). Opioids are usually administered when NSAIDs and local anesthesia are not sufficient to relieve RIPM.

Sodium alginate is a polysaccharide that is widely distributed on the cell walls and interstitium of brown algae. Administered in a solution (approved in Japan for gastritis), it coats the surface of the gastrointestinal mucosa in areas of ulceration or erosion, and has the effect of suppressing tissue destruction and bleeding [4]. In Japan it has been used in the clinical setting as an initial analgesic for esophagitis during radiotherapy or chemo-radiotherapy (CRT). The first randomized trial to investigate whether this compound has a preventive effect against severe esophagitis in patients with locally advanced non-small cell lung cancer undergoing CRT is currently ongoing [5]. Its actual effects in patients treated with neck RT/CRT remain unclear.

The purpose of this retrospective study was to investigate the efficacy and tolerability of sodium alginate for RIPM in patients treated with neck irradiation.

\section{Materials and methods}

The patients' medical data were anonymized prior to access and analysis. The institutional review board (Permission number: H29-20) waived the need for written informed consent from study subjects because all potentially patient-identifying information was removed prior to data analysis.

\section{Patients}

A total of 140 patients received neck external beam radiotherapy at the authors' institution between June 2006 and June 2016. During the same period, 32 patients received $5 \%$ sodium alginate solution orally to treat RIPM; these patients were enrolled in this study. Among the remaining 108 patients, those who were followed up for less than 2 months or did not receive 5\% sodium alginate were excluded from this retrospective study. Table 1 shows the patients' clinical characteristics. The pretreatment evaluation included a complete history, physical examination, complete blood count, body computed tomography and, in some cases, ${ }^{18}$ F-FDG
Table 1 Patient characteristics $(n=32)$

\begin{tabular}{ll}
\hline & $\mathrm{n}(\%)$ \\
\hline Gender & \\
Male & $15(47)$ \\
Female & $17(53)$ \\
Age (median, range) & $66,34-84$ \\
Performance status & \\
O/1/2/3/4 & $7 / 14 / 7 / 3 / 1$ \\
Primary site & \\
Lung & $11(34)$ \\
Breast & $10(31)$ \\
Head and neck & $7(22)$ \\
Other & $4(13)$ \\
Pathology & \\
Squamous cell carcinoma & $11(34)$ \\
Ductal carcinoma & $9(28)$ \\
Adenocarcinoma & $4(13)$ \\
Small cell carcinoma & $2(6)$ \\
Others & $3(9)$ \\
Unknown & $3(9)$ \\
Radiation dose (Gy) & \\
Median (range) & \\
Radiation intent & \\
Radical & \\
Semi-radical & $10(15-72)$ \\
Postoperative & $8 / 24$ \\
Chemotherapy (Yes/No) & \\
& \\
\hline
\end{tabular}

PET/CT and/or magnetic resonance imaging and/or bone scintigraphy.

\section{Sodium alginate administration}

All patients who received 5\% sodium alginate solution took it orally before every meal after the occurrence of RIPM. RIPM was graded as an acute toxicity according to the Common Terminology Criteria for Adverse Events (CTCAE) version 4. The administration of sodium alginate was started when patients were diagnosed with Grade 2 pharyngeal mucositis. In principle, patients took $15 \mathrm{ml}$ of $5 \%$ sodium alginate solution orally. In cases where it was the considered that the patient was less likely to take this dose of sodium alginate, the patient was encouraged to take only $10 \mathrm{ml}$ of the solution. The administration of sodium alginate was continued until the radiotherapy was completed and after the resolution of odynophagia.

\section{Radiotherapy}

All patients were treated with external RT using a linear accelerator with 4, $10 \mathrm{MV}$ or 5-15 MeV electrons 
(Toshiba PRIMUS linear accelerator equipped with standard multi-leaf collimators). CT images were obtained in 5-mm increments over the region of interest. Three-dimensional conformal RT was performed using the Xio (CMS Japan, Tokyo, Japan) treatment planning system in all patients. The clinical target volume (CTV) was defined as the lesion (gross tumor volume and/or regional lymph nodes, or chest wall) plus a $0.5-1.0-\mathrm{cm}$ margin. The planned target volume (PTV) was defined as the CTV plus $0.5-1.0 \mathrm{~cm}$ for the daily setup variation and respiratory movement. In almost all cases, RT was delivered in once-daily fractions of 1.5-3 Gy (five fractions per week). Two of the patients received hyperfractionated RT (1.2 Gy, b.i.d.).

\section{Evaluation}

The efficacy of sodium alginate (improvement of RIPM) was assessed (grade: I, ineffective; II, moderately effective; III, very effective). Odynophagia was evaluated 1-2 times per week by two radiation oncologists. When sodium alginate was ineffective and additional analgesics were required, we recorded both the details of the analgesics and the duration between the administration of sodium alginate and the administration of additional analgesics. We also evaluated the PTV between patients receiving additional analgesics and those who did not.

\section{Statistical analysis}

The relationships between the presence or absence of additional analgesics and the radiation dose and the PTV were investigated using Fisher's exact probability test and the Mann-Whitney U test.

\section{Results}

The median duration from the start of irradiation to the administration of sodium alginate was 15 days (range, 5-36 days). Five of the 32 patients required additional analgesics other than sodium alginate. Moreover, the median duration from the start of sodium alginate treatment until the administration of additional analgesics was 8 days (range, 3-17 days). No sodium alginaterelated toxicities were recorded.

The efficacy of sodium alginate (improvement of RIPM) was scored in all patients according to the abovementioned grading system (Table 2). Among the $32 \mathrm{pa}-$ tients, the improvement of RIPM was observed in 29 patients (moderately effective: 22 patients; very effective: 7 patients). Three patients showed no improvement. There was no difference in the degree of odynophagia between the patients receiving $15 \mathrm{ml}$ of sodium alginate solution and those receiving $10 \mathrm{ml}(p=0.30)$.

Figure 1 shows the relationships between the presence or absence of additional analgesics and the radiation dose. The radiation dose of the patients who required
Table 2 Improvement of RIPM ${ }^{a}$ after administration of sodium alginate $^{b}$

\begin{tabular}{ll}
\hline Efficacy of sodium alginate & Patients (n) \\
\hline II & 3 \\
III & 22 \\
\hline
\end{tabular}

${ }^{\mathrm{a}} \mathrm{RIPM}$; radiation-induced pharyngeal mucositis

bineffective, I; moderately effective, II; very effective, III

additional analgesics was significantly higher than that in the patients who received sodium alginate alone (63.6 \pm 7.8 Gy vs $48.3 \pm 14.8$ Gy, $P=0.02$ ). Regarding the presence or absence of additional analgesics, we also compared the patients who received $>50$ Gy to those who received $\leq 50$ Gy. The patients who received $>50$ Gy tended to require additional analgesics more frequently than those who received $\leq 50$ Gy (Table 3 ). We also evaluated a potential association between the presence or absence of additional analgesics and the PTV, however, there was no significant correlation $\left(192.8 \pm 176.6 \mathrm{~cm}^{3}\right.$ vs $\left.220.5 \pm 164.3 \mathrm{~cm}^{3}, P=0.74\right)$. Incidentally, there was no significant association between the concomitant use of chemotherapy and the need for additional analgesics $(P=0.27)$.

\section{Discussion}

Radiotherapy is often used to treat the neck area in cases of head and neck cancer and after surgery for breast cancer. In head and neck cancer, its role is particularly important from the viewpoint of laryngeal preservation of function and form. However, since the the mucous membrane of the pharyngolarynx is located within the irradiation field, RIPM is almost inevitable. The severity can be aggravated and enhanced when chemotherapy and radiotherapy are administered concurrently. If RIPM occurs, it becomes difficult to eat by mouth; thus intravenous feeding, gastrostomy, or interruption of treatment may be required. Currently, most physicians treat RIPM with analgesics (mainly NSAIDs). Worldwide, NSAIDs are among the most commonly used treatments [6]. Due to their anti-inflammatory and analgesic properties, NSAIDs are often used for pain relief; however, they are associated with upper [7] and lower [8,9] gastrointestinal harm, acute renal failure $[10,11]$ and congestive heart failure [12, 13]. Serious adverse events are rare, but they may not be reversible and can be life-threatening. If these toxicities occur during treatment, they can lead to treatment cessation, which might result in a worse prognosis. The common adverse effects of opioids include nausea, constipation, dizziness, vomiting, sleepiness, pruritus, anorexia and dysuria [14].

To the best of our knowledge, few studies have reported on sodium alginate treatment in patients with RIPM. Sodium alginate is extracted from brown algae, it 


\section{$\mathrm{p}=0.02$}

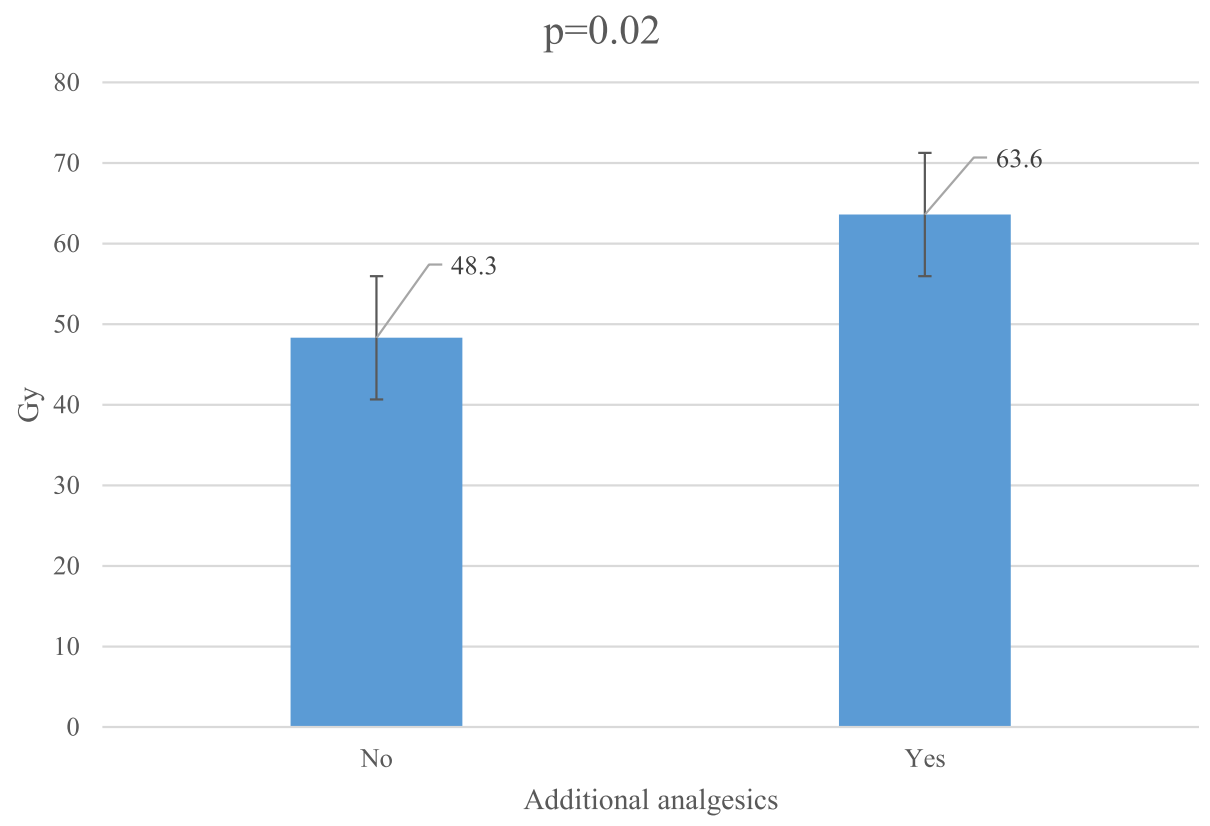

Fig. 1 Relationship between the presence or absence of additional analgesics and the radiation dose

adheres to and protects the surface of the mucosa at sites of erosion. Moreover, it is said to act on the blood coagulation system to have a hemostatic effect and promote wound healing $[4,15]$. In our study, an improvement of RIPM was observed in 29 of the 32 patients $(>90 \%$; moderately effective, $n=22$; very effective, $n=7)$; no adverse events were reported. Oshitani et al. analyzed the effect of sodium alginate in 17 patients with radiotherapy-associated oral mucositis and retrospectively compared them with 22 patients who did not receive sodium alginate treatment. Sodium alginate significantly reduced the pain and erosion of the oral mucosa, which occurred in association with radiotherapy. Similarly to the results of our study, no obvious adverse events occurred in association with the administration of sodium alginate [16]. The results of their research supports the results of our study. In the current study, while good control of RIPM was obtained with sodium alginate, a limit was also observed; specifically, 16\% (5/32) of the patients required additional analgesics other than sodium alginate. In our study, the

Table 3 Use of additional analgesics according to the dose of radiation

\begin{tabular}{lllllll}
\hline & & \multicolumn{2}{l}{ Additional analgesics } & & \\
\cline { 3 - 4 } & & Yes & No & & Total & $p^{*}$ \\
\hline Radiation dose & $<50$ Gy & 4 & 10 & & 14 & 0.10 \\
& 250 Gy & 1 & 17 & & 18 & \\
Total & & 5 & 27 & & 32 & \\
\hline
\end{tabular}

*Fisher's exact test radiation dose of the patients who required additional analgesics was significantly higher than that of the patients who received sodium alginate alone $(63.6 \pm 7.8$ Gy vs $48.3 \pm 14.8 \mathrm{~Gy}, P=0.02$ ). Moreover, the patients who received a radiation dose of $>50$ Gy tended to require additional analgesics more frequently than those who received a radiation dose of $\leq 50 \mathrm{~Gy}$. Based on the above-mentioned findings, sodium alginate could be an ideal analgesic for the management of RIPM in cancer patients treated with neck irradiation; however, we believe that it is necessary to properly use additional NSAIDs or opioids in patients receiving radiation doses of $\geq 50$ Gy. Our results demonstrated that the administration of sodium alginate is a promising supportive therapy that can be administered without fear of side effects in patients treated with neck irradiation. Treatment protocols using sodium alginate not only allow a reduction in the use of NSAIDs and opioids, but are also expected to reduce medical expenses.

\section{Conclusion}

The present study is associated with several limitations. First, the study population was relatively small, and the study was conducted retrospectively in a single institute. Furthermore, both the patient profiles and RT regimens were heterogeneous. Additionally, only the patients who received sodium alginate were analyzed; thus, the potential for a selection bias should be considered. It is also necessary to further investigate whether concomitant chemotherapy is associated with RIPM. Thus, in the future, a large prospective randomized control study 
should be performed to investigate the actual benefits of sodium alginate for RIPM.

In summary, this is the first report that has attempted to assess the feasibility of sodium alginate administration in the treatment of RIPM in patients undergoing neck irradiation. Sodium alginate was found to be effective and well tolerated. The RT dose was found to be significantly correlated with the need for additional analgesics. These results justify further prospective evaluations with detailed treatment protocols, including studies investigating the radiation dose and fractionation, in order to clarify whether sodium alginate can improve RIPM.

\section{Abbreviations}

CRT: Chemo-radiotherapy; CTCAE: Common Terminology Criteria for Adverse Events; CTV: Clinical target volume; NSAIDS: Nonsteroidal anti-inflammatory drugs; PTV: Planned target volume; RIPM: Radiation-induced pharyngeal mucositis; RT: Radiotherapy

\section{Acknowledgements}

The author gratefully acknowledges all staff members of Radiology, Kitakyushu General Hospital.

\section{Funding}

No funding received.

\section{Availability of data and materials}

The datasets analyzed during the current study are available from the corresponding author upon reasonable request.

\section{Authors' contributions}

SY collected and analyzed data, and drafted the manuscript. The author read and approved the final manuscript

\section{Ethics approval and consent to participate}

The author declares no conflicts of interest associated with this manuscript. The patients' medical data were anonymized prior to access and analysis. The institutional review board (Permission number: H29-20) waived the need for written informed consent from study subjects because all potential patient-identifying information was removed prior to data analysis.

\section{Consent for publication}

Not applicable. Manuscript does not contain any individual person's data.

\section{Competing interests}

The authors declare that they have no competing interests.

\section{Publisher's Note}

Springer Nature remains neutral with regard to jurisdictional claims in published maps and institutional affiliations.

Received: 24 October 2018 Accepted: 7 April 2019

Published online: 23 April 2019

\section{References}

1. Uncan W, MacDougall H, Kerr G, et al. The adverse effect of treatment gaps in the outcome of radiotherapy for laryngeal cancer. Radiother Oncol. 1996; $41 \cdot 203-7$

2. Robertson $\mathrm{C}$, Robertson AG, Hendry $\mathrm{JH}$, et al. Similar decreases in local tumor control are calculated for treatment protraction and for interruptions in the radiotherapy of carcinoma of the larynx in four centers. Int J Radiat Oncol Biol Phys. 1998:40:319-29.

3. Kwong DL, Sham JS, Chua DT, et al. The effect of interruptions and prolonged treatment time in radiotherapy for nasopharyngeal carcinoma. Int J Radiat Oncol Biel Phvs. 1997;39:703-10.
4. Daigo K, Wada Y, Yamada C, et al. Pharmacological studies of sodium alginate (I): protective effect of sodium alginate on mucous membranes of uppergastrointestinal tract [in Japanese]. Yakugaku Zasshi. 1981;101:452-7.

5. Ninomiya K, Ichihara E, Hotta K, et al. Three-Arm Randomized Trial of Sodium Alginate for Preventing Radiation-Induced Esophagitis in Locally Advanced Non-Small Cell Lung Cancer Receiving Concurrent Chemoradiotherapy: The OLCSG1401 Study Protocol. Clin Lung Cancer. 2017;18:245-9.

6. Warner TD, Giuliano F, Vojnovic I, et al. nonsteroid drug selectivities for cyclo-oxygenase-1 rather than cyclo-oxygenase-2 are associated with human gastrointestinal toxicity: a full in vitro analysis. Proc Natl Acad Sci U S A. 1999;96:7563-8.

7. Hernández-Diaz S, García Rodriguez LA. Association between nonsteroidal anti-inflammatory drugs and upper gastrointestinal tract bleeding and perforation: An overview of epidemiological studies published in the 1990s. Arch Intern Med. 2000;160:2093-9.

8. Allison MC, Howatson AG, Torrance CJ, et al. Gastrointestinal damage associated with the use of nonsteroidal antiinflammatory drugs. N Engl J Med. 1992;327:749-54

9. Lanas A, Serrano P, Bajador E, et al. Evidence of aspirin use in both upper and lower gastrointestinal perforation. Gastroenterology. 1997;112:683-9.

10. Henry D, Page J, Whyte I, et al. Consumption of nonsteroidal antiinflammatory drugs and the development of functional renal impairment in elderly subjects. Results of a case-control study. Br J Clin Pharmacol. 1997;44: 85-90.

11. Griffin MR, Yared A, Ray WA, et al. Nonsteroidal antiinflamatory drugs and acute renal failure in elderly persons. Am J Epidemiol. 2000;151:488-96.

12. Page J, Henry D. Consumption of NSAIDs and the development of congestive heart failure in elderly patients: An underrecognized public health problem. Arch Intern Med. 2000;160:777-84.

13. Garcia Rodriguez LA, Hernandez-Diaz S. Non-steroidal anti-inflammatory drugs as a trigger of clinical heart failure. Epidemiology. 2003;14:240-6.

14. Wang YM, Liu ZW, Liu JL, et al. Efficacy and tolerability of oxycodone in moderate-severe cancer-related pain: a meta-analysis of randomized controlled trials. Exp Ther Med. 2012:4:249-54.

15. Daigo K, Yamada C, Wada Y, et al. Pharmacological studies of sodium alginate. II. Hemostatic effect of sodium alginate on gastrointestinal bleeding [Article in Japanese]. Yakugaku Zasshi. 1981;101:458-63.

16. Oshitani T, Okada K, Kushima T, et al. Clinical evaluation of sodium alginate on oral mucositis associated with radiotherapy [Article in Japanese]. Nihon Gan Chiryo Gakkai Shi. 1990;25:1129-37.

Ready to submit your research? Choose BMC and benefit from:

- fast, convenient online submission

- thorough peer review by experienced researchers in your field

- rapid publication on acceptance

- support for research data, including large and complex data types

- gold Open Access which fosters wider collaboration and increased citations

- maximum visibility for your research: over $100 \mathrm{M}$ website views per year

At BMC, research is always in progress.

Learn more biomedcentral.com/submissions 